\title{
THE FIRST THREE LARGEST NUMBERS OF SUBUNIVERSES OF SEMILATTICES
}

\author{
DELBRIN AHMED AND ESZTER K. HORVÁTH
}

Received 03 June, 2020

\begin{abstract}
Let $(L, \vee)$ be a finite n-element semilattice where $n \geq 5$. We prove that the first largest number of subuniverses of an $n$-element semilattice is $2^{n}$, while the second largest number is $28 \cdot 2^{n-5}$ and the third one is $26 \cdot 2^{n-5}$. Also, we describe the $n$-element semilattices with exactly $2^{n}, 28 \cdot 2^{n-5}$ or $26 \cdot 2^{n-5}$ subuniverses.

2010 Mathematics Subject Classification: 06A12; 06B99
\end{abstract}

Keywords: finite lattice, sublattice, subuniverse, finite semilattice, number of subuniverses

\section{INTRODUCTION AND OUR RESULT}

For a semilattice $(L, \vee), \operatorname{Sub}(L, \vee)$ will denote its subuniverse-lattice. By a subuniverse, we mean a subsemilattice or the emptyset. All semilattices occurring in this paper will be assumed to be finite. On a semilattice $(L, \leq)$, we have a natural partial ordering defined by

$$
x \leq y \Longleftrightarrow x \vee y=y .
$$

Conversely, if $(L, \leq)$ is partial order in which any two elements $x, y$ have a least upper bound $x \vee y$, then $(L, \vee)$ is a semilattice. For any $x, y$ in a join-semilattice, $x \wedge y$ is defined by their infimum provided it exists; if this infimum does not exist, then $x \wedge y$ is undefined. Let $P$ and $Q$ be posets with disjoint underlying sets. Then the ordinal sum $P+_{\text {ord }} Q$ is the poset on $P \cup Q$ with $s \leq t$ if either $s, t \in P$ and $s \leq t$; or $s, t \in Q$ and $s \leq t$; or $s \in P$ and $t \in Q$. To draw the Hasse diagram of $P+_{\text {ord }} Q$, we place the Hasse diagram of $Q$ above that of $P$ and then connect any minimal element of $Q$ with any maximal element of $P$; see Figure 1 . If $K$ with 1 and $L$ with 0 are finite posets, then their glued sum $K+$ glu $L$ is the ordinal sum of the posets $K \backslash\left\{1_{K}\right\}$, the singleton poset, and $L \backslash\left\{0_{L}\right\}$, in this order; see Figure 2. Note that $+_{\text {glu }}$ is an associative but not a commutative operation.

The semilattices $H_{3}$ and $H_{4}$ will be used later, see Figure 3.

This research was supported by grant TUDFO/47138-1/2019-ITM of the Ministry for Innovation and Technology, Hungary. This research of the second author was partially supported by NFSR of Hungary (OTKA), grant number K 115518. 

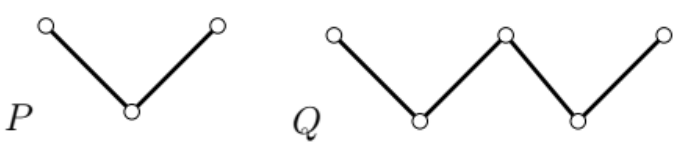

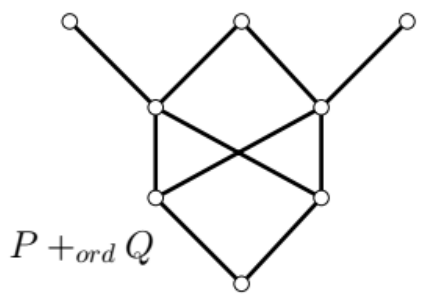

Figure 1. The ordinal sum $P+{ }_{\text {ord }} Q$ of $P$ and $Q$
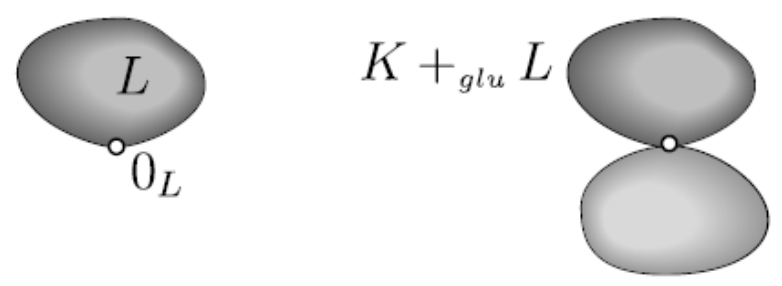

FIGURE 2. The glued sum $K+{ }_{\text {glu }} L$ of $K$ and $L$

Our result is motivated by similar results, see Ahmed and Horváth [1], Czédli [3-7] and Czédli and Horváth [8]. To obtain more information about lattice theory and semilattices we direct the reader to the bibliography indicated in $[9,10]$ and [2], respectively.

For a natural number $n \in \mathbb{N}^{+}:=\{1,2,3, \ldots\}$, let

$$
\operatorname{NS}(n):=\{|\operatorname{Sub}(L)|: L \text { is a semilattice of size }|L|=n\} .
$$

Theorem 1. If $5 \leq n \in \mathbb{N}^{+}$, then the following assertions hold.

(i) The largest number in $\mathrm{NS}(n)$ is $2^{n}=32 \cdot 2^{n-5}$. Furthermore, an n-element semilattice $(L, \vee)$ has exactly $2^{n}$ subuniverses if and only if $(L, V)$ is a chain.

(ii) The second largest number in $\mathrm{NS}(n)$ is $28 \cdot 2^{n-5}$. Furthermore, an n-element semilattice $(L, \vee)$ has exactly $28 \cdot 2^{n-5}$ subuniverses if and only if $(L, \vee) \cong$ $H_{3}+{ }_{\mathrm{glu}} C_{1}$ or $(L, \vee) \cong C_{0}+{ }_{\text {ord }} H_{3}+{ }_{\mathrm{glu}} C_{1}$, where $C_{0}$ and $C_{1}$ are finite chains.

(iii) The third largest number in $\mathrm{NS}(n)$ is $26 \cdot 2^{n-5}$. Furthermore, an n-element semilattice $(L, \vee)$ has exactly $26 \cdot 2^{n-5}$ subuniverses if and only if $(L, \vee) \cong$ $H_{4}+\mathrm{glu} C_{1}$ or $(L, \vee) \cong C_{0}+{ }_{\text {ord }} H_{4}+\mathrm{glu} C_{1}$, where $C_{0}$ and $C_{1}$ are finite chains.

\section{TWO PREPARATORY LEMMAS}

An element $u$ of a semilattice $L$ is called a narrow element, or a narrows for short, if $u \neq 1_{L}$ and $L=\uparrow u \cup \downarrow u$. That is, if $u \neq 1_{L}$ and $x \| u$ holds for no $x \in L$. 
The notion of a binary partial algebra is well known, but the reader can refresh his/her knowledge from [4]. Let $\mathcal{A}$ be a finite $n$-element binary partial algebra. A subuniverse of $\mathcal{A}$ is a subset $X$ of $\mathcal{A}$ such that $X$ is closed with respect to all partial operations. The set of subuniverses of $\mathcal{A}$ will be denoted by $\operatorname{Sub}(\mathcal{A})$. The relative number of subuniverses of $\mathcal{A}$ denoted by $\sigma_{k}(\mathcal{A})$ is defined as follows:

$$
\sigma_{k}(\mathcal{A})=|\operatorname{Sub}(\mathcal{A})| \cdot 2^{k-n}
$$

In order to comply with [8], will use $k=5$. The original definition of $\sigma_{k}$ is given in the paper of Czédli [4], there he used $k=8$.

Lemma 1. If $(K, \vee)$ is a subsemilattice and $H$ is a subset of a finite semilattice $(L, V)$, then the following three assertions hold.

(i) With the notation $t:=|\{H \cap S: S \in \operatorname{Sub}(L, \vee)\}|$, we have that

$$
\sigma_{k}(L, \vee) \leq t \cdot 2^{k-|H|} \text {. }
$$

(ii) $\sigma_{k}(L, \vee) \leq \sigma_{k}(K, \vee)$.

(iii) Assume, in addition, that $(K, \vee)$ has no narrows. Then $\sigma_{k}(L, \vee)=\sigma_{k}(K, \vee)$ if and only if $(L, \vee)$ is (isomorphic to) $C_{0}+{ }_{\text {ord }}(K, \vee)+\mathrm{glu} C_{1}$, where $C_{1}$ is a chain, and $C_{0}$ is a chain or the emptyset.

Proof. Parts (i) and (ii) can be extracted from the proof of in Lemma 2.3 of [4]. The argument there yields a bit more than stated in (i) and (ii); namely, for later reference, note the following.

If $\sigma_{k}(L, \vee)=\sigma_{k}(K, \vee)$, then for every $S \in \operatorname{Sub}(K, \vee)$ and every subset $X$ of $L \backslash K$ we have that $S \cup X \in \operatorname{Sub}(L, \vee)$.

Next, to prove part (iii), let $n:=|(L, \vee)|$ and $m:=|(K, \vee)|$. Let $k:=5$, the case of another $k$ is analogous. Assume that $(K, \vee)$ has no narrows. First, let $(L, \vee)=$ $C_{0}+_{\text {ord }}(K, \vee)+$ glu $C_{1}$. It is obvious that whenever $X \subseteq L \backslash K$ and $S \in \operatorname{Sub}(K, \vee)$, then $S \cup X \in \operatorname{Sub}(L, \vee)$. Since $L \backslash K$ has $2^{|L|-|K|}$ subsets, $|\operatorname{Sub}(L, \vee)| \geq|\operatorname{Sub}(K, \vee)|$. $2^{|L|-|K|}$. Dividing this inequality by $2^{n-5}=2^{m-5} \cdot 2^{|L|-|K|}$ we obtain the required equality, as the converse inequality given in part (ii).

Conversely, assume the equality in (iii). We claim that

$$
\text { for all } y \in K \text { and for all } x \in L \backslash K, y \nVdash x \text {. }
$$

Suppose the contrary. If $y \in K$, then $\{y\} \in \operatorname{Sub}(K)$. If $x \in L \backslash K$ and $y \| x$, then $\{y, x\}$ is not a subuniverse of $L$, contradicting (2.1).

We claim that

$$
\text { for all } x \in L \backslash K, x \ngtr 1_{K} \text { implies that for all } y \in K, x<y \text {. }
$$

Suppose the contrary and pick $x$ in $L \backslash K$ and $y \in K$ such that $x \ngtr 1_{K}$ and $x \nless y$. Using (2.2) and $x \neq y$, we have that $y<x<1_{K}$. Let $p:=\bigvee\{s \in K: s<x\}$; this exists by finiteness and $y \leq p \leq x$. In fact, $p \in K$ as $K$ is a subsemilattice of $L$ but $x \notin K$, so $y \leq p<x$. Now let $u \in K$ such that $u \not \leq p$. We know from (2.2) that $u \nmid x$. 
If we had $u \leq x$ (in fact, $u<x$ since $x \notin K$ ), then $u$ would be one of the joinands defining $p$ and so $u \leq p$ would be a contradiction. Hence $x<u$, and so $p<x<u$ implies $p<u$. We have seen that, for any $u \in K, u \not \leq p$ implies $p<u$. In other words, $K=\uparrow_{K} p \cup \downarrow_{K} p$, which means $p$ is a narrows, contradicting our assumption on $K$. Thus, (2.3) holds. Finally, we show that $L \backslash K$ is a chain. Indeed, if $L \backslash K$ is not a chain, say $a \| b, a \in L \backslash K$ and $b \in L \backslash K$, then $\varnothing \in \operatorname{Sub}(K)$ extended by $\{a, b\} \notin \operatorname{Sub}(L)$ would contradict (2.1). Define $C_{1}=\left\{x \in L \backslash K: x \geq 1_{K}\right\}$; it is a chain (a subchain of $L \backslash K)$. Let $C_{0}=(L \backslash K) \backslash C_{1}$; it is either a chain or empty. If $C_{0}$ is empty, then $L$ is $K+{ }_{\text {glue }} C_{1}$, as required. If $C_{0}$ is nonempty, then its elements are less than any element of $K$ by (2.3), and so $L=C_{0}+{ }_{\text {ord }} K+_{\text {glue }} C_{1}$, as required.

The following lemma can be proved by a computer program, but for the reader's convenience, we give its proof.

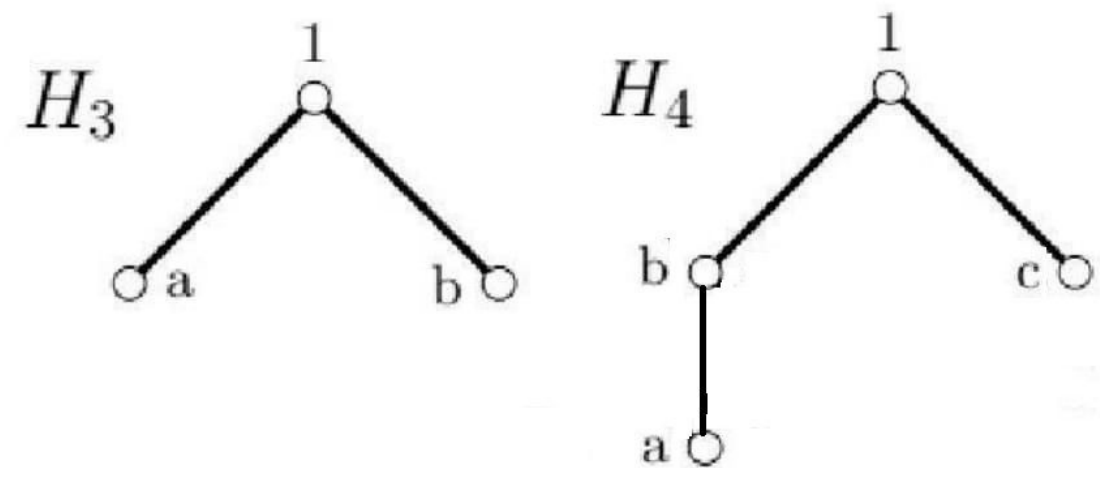

FIGURE $3 . H_{3}$ and $H_{4}$

Lemma 2. For the join-semilattices given in Figure 3 the following assertions hold.

(i) $\sigma_{5}\left(H_{3}\right)=28$,

(ii) $\sigma_{5}\left(H_{4}\right)=26$,

Proof. The notations given by Figure 3 will be used. For later reference, note that if $(L, \vee)$ is a chain then $|S u b(L, \vee)|=2^{|(L, V)|}$.

For (i), observe that

$$
\begin{aligned}
& \left|\left\{S \in \operatorname{Sub}\left(H_{3}, \vee\right): a \notin S\right\}\right|=4, \quad \text { (S is chain), } \\
& \left|\left\{S \in \operatorname{Sub}\left(H_{3}, \vee\right): a \in S,\{b\} \cap S=\varnothing\right\}\right|=2, \text { and } \\
& \left|\left\{S \in \operatorname{Sub}\left(H_{3}, \vee\right): a \in S,\{b\} \cap S \neq \varnothing\right\}\right|=1,
\end{aligned}
$$

whereby $\left|\operatorname{Sub}\left(H_{3}, \vee\right)\right|=4+2+1=7=28 \cdot 2^{3-5}$, note that $\sigma_{5}\left(H_{3}\right)=28$ proves (i). 
For (ii), let us compute

$$
\begin{aligned}
& \left|\left\{S \in \operatorname{Sub}\left(H_{4}, \vee\right): c \notin S\right\}\right|=7, \quad \text { by (i), } \\
& \left|\left\{S \in \operatorname{Sub}\left(H_{4}, \vee\right): c \in S,\{a, b\} \cap S=\varnothing\right\}\right|=2, \text { and } \\
& \left|\left\{S \in \operatorname{Sub}\left(H_{4}, \vee\right): c \in S,\{a, b\} \cap S \neq \varnothing\right\}\right|=4 .
\end{aligned}
$$

Hence, $\left|\operatorname{Sub}\left(H_{4}, \vee\right)\right|=7+2+4=13=26 \cdot 2^{4-5}$, while $\sigma_{5}\left(H_{4}\right)=26$ proves (ii).

Remark 1. For counting subsemilattices, a computer program is available on G. Czédli's webpage: http://www.math.u-szeged.hu/czedli/

\section{THE REST OF THE PROOF}

Proof of Theorem 1. Part (i) is trivial. For part (ii) let $(L, \vee)$ be an $n$-element semilattice. We know from Lemma 1 (iii) that if

$$
(L, \vee) \cong H_{3}+{ }_{\mathrm{glu}} C_{1} \text { or }(L, \vee) \cong C_{0}+{ }_{\text {ord }} H_{3}+{ }_{\mathrm{glu}} C_{1} \text {, where } C_{0} \text { and } C_{1} \text { are chains, }
$$

then $\sigma_{5}(L)=\sigma_{5}\left(H_{3}\right)=28$, indeed. Conversely, assume that $\sigma_{5}(L)=28$. Then it follows from part (i) that $L$ is not a chain. So $L$ has two incomparable elements, $a$ and $b$. Clearly, $\{a, b, a \vee b\}$ is a join-subsemilattice isomorphic to $H_{3}$. But $\sigma_{5}\left(H_{3}\right)$ is also 28 by Lemma 2 (i). Thus, Lemma 1 (iii) immediately yields that $L$ is of the desired form. By this we completed the proof of part (ii) of Theorem 1.

We prove part (iii).

Assume that $(L, \vee)$ is of the given form, then $\sigma_{5}(L, \vee)=26$ is clear from Lemma 2 (ii) and Lemma 1 (iii). In order to prove the converse, that is, the nontrivial implication, assume that $\sigma_{5}(L, \vee)=26$. By Theorem 1 (i), $(L, \vee)$ has two incomparable elements, $a$ and $b$. By part Theorem 1 (ii), $\{a, b\}$ is not the only 2-element antichain in $L$ since otherwise $\sigma_{5}(L, \vee)$ would be 28 . To complete the proof, consider the following cases.

Case 1: There is an antichain $\{c, d\}$ disjoint from $\{a, b\}$, where the elements $a, b, c, d$ are distict. Let $x:=a \vee b$ and $y:=c \vee d$. There are cases depending on $t:=|\{a, b, c, d, x, y\}|$, which is 4,5 , or 6 . The number of possible cases can be reduced by symmetry: $a$ and $b$ play a symmetric role, so do $c$ and $d$, and so do $\{a, b\}$ and $\{c, d\}$ and thus $\mathrm{x}$ and $\mathrm{y}$. We consider three sub-cases as we will see below:

Sub-case 1a: Now $t=6$. Take the partial algebra $U_{1}=\{a, b, c, d, x, y\}$ with $a \vee b=$ $x$ and $c \vee d=y$. This six-element partial algebra has $\sigma_{5}\left(U_{1}\right)=24.5$, this can be checked by the mentioned computer program. By Lemma 2.3 from [4] we obtain that $\sigma_{5}(L) \leq \sigma_{5}\left(U_{1}\right) \leq 24.5$, contradicting $\sigma_{5}(L)=26$. Thus, this case is excluded.

Sub-case 1b: Now $t=5$. By symmetry, $y=c \vee d$ is not a new element, so $y=c \vee d$ is either $x$ or $a$. The case $y=b$ need not be considered because $a$ is symmetric to $b$. Therefore, this sub-case $1 \mathrm{~b}$ is split in two cases as follows: 
First, where $y=x$, this case is captured by taking the partial algebra $U_{2}=$ $\{a, b, c, d, x\}$ with $a \vee b=x, c \vee d=x$. It has $\sigma_{5}\left(U_{2}\right)=25$ by the mentioned computer program. Like above, this implies $\sigma_{5}(L) \leq \sigma_{5}\left(U_{2}\right) \leq 25$, contradicting $\sigma_{5}(L)=26$.

Second, where $y=a$, this case is captured by taking the partial algebra $U_{3}=$ $\{a, b, c, d, x\}$ with $a \vee b=x, c \vee d=a$. This five-element partial algebra has $\sigma_{5}\left(U_{3}\right)=$ $24<26$, and we get a contradiction as above.

By the above we can note that the sub-case $1 \mathrm{~b}$ is excluded since so are both of its subcases.

Sub-case 1c: Here $t=4$. Then $x=a \vee b$ is one of $c$ and $d$. By symmetry, we can assume that $a \vee b=c$. However, then $a<c<c \vee d, b<c<c \vee d$, whereby $y=c \vee d$ is none of the elements $a, b, c, d$, contradicting $t=4$. So this case is excluded.

After having all of its sub-cases excluded, we obtain that Case 1 is excluded. That is, no two-element antichain is disjoint from $\{a, b\}$. But remember that there is another two-element antichain, whereby, by a-b symmetry, we consider Case 2: there is an element $c$ such that $a$ and $c$ are incomparable. Again there are two sub-cases according to the position of $b$ and $c$.

Sub-case 2a: Here $b$ and $c$ are also incomparable. Here, we have to investigate, how many of $a \vee b, a \vee c$, and $b \vee c$ are equal to $a \vee b \vee c$. Since the answer could be $0,1,2$ or 3 (and using symmetry), it suffices to consider only the following four join-semilattices. The first join-semilattice is $K_{0}=\{a, b, c, z, x, y, 1\}$ with edges $a x$, $b x, b y, c y, a z, c z, x 1, y 1, z 1$ and equalities $a \vee b=x, b \vee c=y, a \vee c=z$; this gives $\sigma_{5}\left(K_{0}\right)=15.25$. The second join-semilattice is $K_{1}=\{a, b, c, x, y, 1\}$ with edges $a x$, $b x$, by, $c y, x 1, y 1$ and equalities $a \vee b=x, b \vee c=y, a \vee c=1$; this gives $\sigma_{5}\left(K_{1}\right)=18.5$. The third is $K_{2}=\{a, b, c, x, 1\}$ with edges $a x, b x, x 1, c 1$ and constraints $a \vee b=x$, $a \vee c=1, b \vee c=1$; this gives $\sigma_{5}\left(K_{2}\right)=22$. The fourth is $K_{3}=\{a, b, c, 1\}$ with edges $a 1, b 1, c 1$, and equalities $a \vee b=1, a \vee c=1, b \vee c=1$; this gives $\sigma_{5}=24$. Since one of $K_{0}, K_{1}, K_{2}$, and $K_{3}$ is a subsemilattice of $L$ and all the four $\sigma_{5}$ values of these join-semilattices are smaller than 26 , therefore sub-case $2 \mathrm{a}$ is excluded.

Sub-case 2b: Here $b$ and $c$ are comparable in addition to that $a$ and $c$ are incomparable and so do $a$ and $b$. At present, $b$ and $c$ play a symmetric role. So we can assume that $b<c$. Suppose, for contradiction, that $x:=a \vee b<a \vee c=: 1$. By the incomparabilities assumed, $|\{a, b, c, x, 1\}|=5$; for example if $x=a \vee b=c$ is impossible since it would yield $a<c$. The mentioned constraints are defining $K$. Now $\sigma_{5}(K)=23<26$ gives a contradiction. So $a \vee b<a \vee c$ fails but $a \vee b \leq a \vee c$ since $b<c$. Therefore, with $1=a \vee b=a \vee c,\{a, b, c, 1\}$ is a subsemilattice (isomorphic to) $H_{4}$.

Now that all other possibilities have been excluded, we know that $H_{4}$ is a joinsubsemilattice of $(L, \vee)$. Observe that $H_{4}$ has no narrows. Therefore, by Lemma 1 (iii), $(L, \vee)$ is of the desired form. By this, the proof of Theorem 1 is completed. 


\section{REFERENCES}

[1] D. Ahmed and E. K. Horváth, "Yet two additional large numbers of subuniverses of finite lattices," Discussiones Mathematicae - General Algebra and Applications, vol. 39, no. 2, p. 251, 2019, doi: 10.7151/dmgaa.1309.

[2] I. Chajda, R. Halaš, and J. Kühr, Semilattice structures. Heldermann Lemgo, 2007, vol. 30.

[3] G. Czédli, "A note on finite lattices with many congruences," Acta Univ. M. Belii Ser. Math., pp. 22-28, 2018.

[4] G. Czédli, "Eighty-three sublattices and planarity," Algebra universalis, vol. 80, no. 4, p. 45, 2019, doi: 10.1007/s00012-019-0615-3.

[5] G. Czédli, "Finite semilattices with many congruences," Order, vol. 36, no. 2, pp. 233-247, 2019, doi: 10.1007/s11083-018-9464-5.

[6] G. Czédli, "Lattices with many congruences are planar," Algebra universalis, vol. 80, no. 1, p. 16, 2019, doi: 10.1007/s00012-019-0589-1.

[7] G. Czédli, "One hundred twenty-seven subsemilattices and planarity," Order, pp. 1-11, 2019, doi: /10.1007/s11083-019-09519-x.

[8] G. Czédli and E. K. Horváth, "A note on lattices with many sublattices," Miskolc Mathematical Notes, vol. 20, no. 2, p. 839-848, 2019, doi: 10.18514/MMN.2019.2821.

[9] G. Grätzer, Lattice theory: foundation. Springer Science \& Business Media, 2011.

[10] I. Rival and R. Wille, "Lattices freely generated by partially ordered sets: which can be" drawn"?" Journal für die reine und angewandte Mathematik, vol. 1979, no. 310, pp. 56-80, 1979, doi: 10.1515/crll.1979.310.56.

Authors' addresses

Delbrin Ahmed

University of Szeged, Bolyai Institute, Aradi vértanúk tere 1, 6720 Szeged, Hungary

E-mail address: delbrin@math.u-szeged.hu

Eszter K. Horváth

(Corresponding author) University of Szeged, Bolyai Institute, Aradi vértanúk tere 1, 6720 Szeged, Hungary

E-mail address: horesztemath.u-szeged.hu 